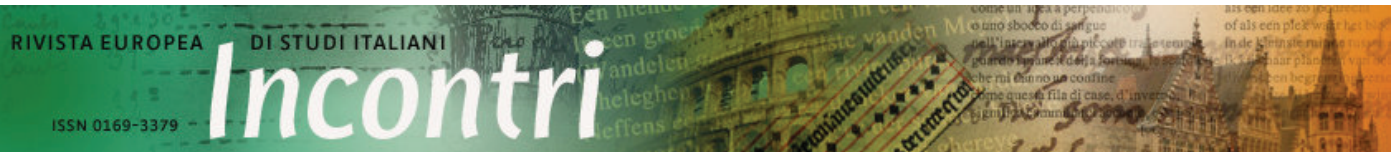

URN:NBN:NL:UI:10-1-114239 - Publisher: Igitur publishing

Content is licensed under a Creative Commons Attribution 3.0 License

Anno 27, 2012 / Fascicolo 2 - Website: www.rivista-incontri.nl

\title{
Quale filosofia per la vita? Le scomode eredità di un puro metafisico
}

\author{
Recensione di: Federico Luisetti, Una vita. Pensiero selvaggio e \\ filosofia dell'intensità, Milano-Udine, Mimesis, 2011, 158 p., ISBN: \\ 978-88-5750-618-0, € 14, 00.
}

\section{Fabio Treppiedi}

Affacciatasi nel dibattito contemporaneo sul finire degli anni Settanta con Foucault, la biopolitica si concentra sulla trasformazione dei rapporti tra vita e politica: se ad esempio nell'antichità l'uomo era considerato 'un animale vivente ed inoltre capace di esistenza politica', nella modernità l'uomo diventa 'un animale nella cui politica è in questione la sua vita di essere vivente'. ${ }^{1}$ Foucault inscrive la biopolitica in una concreta rete storica, il biopotere, consolidatasi col liberalismo: le 'strategie' della biopolitica non esulano infatti dalle 'pratiche' attraverso cui i governi disciplinano e controllano le popolazioni. Ridefinendo la transizione dall'antichità alla modernità, Agamben rintraccia le radici della biopolitica nella Politica di Aristotele. Da qui emergono due termini chiave della biopolitica: la zoé, 'il semplice fatto di vivere, comune a tutti gli esseri viventi' e il bios, la forma di vita del singolo o del gruppo. ${ }^{2}$

La biopolitica ha pertanto implicato la disamina dialettica dei problemi man mano emersi: così come, per riflettere sui temi della biopolitica (sovranità, comunità, conflitto, norme di vita, ecc.), è opportuno risalire alla differenza tra zoé e bios, risulta opportuno, allo stesso scopo, lavorare sulle contraddizioni più incisive del rapporto tra vita e politica. Esposito propone allora la relazione tra biopolitica e tanatopolitica. Quest'ultima costituirebbe l''anello assente della riflessione foucaultiana' dal momento che la 'genesi specificamente moderna' della biopolitica non emerge a prescindere dall'analisi dei modi in cui, nei totalitarismi del Novecento, il potere stabilisce ordine 'producendo' morte: la 'politicità del bios' si afferma, indica Esposito, nella traduzione immediatamente politica della zoé e, al tempo stesso, nella caratterizzazione intrinsecamente biologica della politica. ${ }^{3}$

La definizione di nuove prospettive biopolitiche implica più in generale, soprattutto oggi, un confronto con le significative voci sopraccennate. Un tale confronto fa da sfondo a Una vita, libro in cui Federico Luisetti ripensa a fondo la biopolitica grazie ad un percorso storico filosofico incentrato da un lato su un vis à vis con Deleuze e, dall'altro, sul recupero in chiave naturalistica del 'vitalismo' di

\footnotetext{
${ }^{1}$ M. Foucault, La volontà di sapere, Milano, Feltrinelli, 1984, p. 127.

${ }^{2} \mathrm{G}$. Agamben, Homo sacer. Il potere sovrano e la nuda vita, Torino, Einaudi, 2005, p. 3.

${ }^{3}$ R. Esposito, Bios. Biopolitica e filosofia, Torino, Einaudi, 2004, p. XIII.
} 
Bergson e sulla declinazione politica dell' 'orientalismo' di Nietzsche. Se Luisetti ci presenta Una vita come un libro su Deleuze, pertanto, lo fa nella misura in cui ci invita ad interrogarci non soltanto sul come il filosofo francese pensa la vita ma anche sul senso in cui l'Occidente l'ha finora pensata. In Una vita ci si chiede se esiste un pensiero della vita 'immediatamente' politico. Luisetti discute da qui le posizioni di Esposito e Agamben, i quali intravedono nell'ultimo scritto di Deleuze, L'immanenza: una vita..., le premesse di un nuovo vitalismo filosofico politico. ${ }^{4}$ Mentre Agamben vi scorge una via per pensare la vita come 'concetto filosoficopolitico-teologico' attraverso cui 'ripensare' l'Occidente intero, ${ }^{5}$ Esposito vi rintraccia una 'biopolitica affermativa' che 'inverte la relazione reciprocamente distruttiva' tra vita e norma spinta all'estremo dal nazismo. ${ }^{6}$ Può davvero il pensiero deleuziano, si domanda però Luisetti, essere considerato una biopolitica 'assolutamente' affermativa? Quanto è 'assoluta' la sua immanenza? Un autentico 'pensiero vivente' immediatamente connesso con l'agire dovrebbe di per sé stesso resistere a tutto ciò che lo 'raggela' e lo 'pietrifica' fino a pregiudicare l'unitarietà del nesso tra vita e azione.

Da 'puro metafisico', ${ }^{7}$ Deleuze non pensa fino in fondo l'immanenza, questa la tesi centrale di Una vita, poiché compromette alla radice l'unitarietà del nesso tra vivere e agire, introducendo l'immobilità nella vita come un che di 'connaturato' al suo movimento. La filosofia di Deleuze è così un 'vitalismo trascendentale' che coglie la vita solo in linea di principio (p. 35). Resta infatti fondamentale, per Deleuze, 'relazionare' la vita a 'condizioni necessarie' che, per così dire, la rendono viva. Un principio, quello cui Deleuze si appella, che risponde contemporaneamente a tre condizioni: si genera nella zoé, non si confonde con la zoé stessa e indica il senso per cui la zoé si individua in 'un' bios. ${ }^{8}$ Luisetti evidenzia quanto la prima esigenza di Deleuze, nel suo 'trascendentalismo dell'impersonale' (p. 10), rimane quella di rendere pensabile la vita. Esigenza sintomatica, in Deleuze come in altri filosofi, dell'abitudine fin troppo occidentale ad assumere la 'vita contemplativa' (l'aristotelico bíos theoretikós) come la 'forma di vita' migliore, 'snaturando' il nesso tra il vivere e l'agire laddove il pensiero 'svuota' la zoé del movimento che più le è proprio (p. 109).

Luisetti mette in luce questo filo conduttore della filosofia di Deleuze insistendo metodicamente sul peso dell'eredità kantiana di Deleuze. Una vita intende infatti mostrare che il filosofo francese non pensa fino in fondo la vita proprio per la sua 'aspirazione kantiana'" e per la sua impostazione 'teoretica' di fondo. Kant rappresenta non a caso il farmakon con cui Deleuze, secondo Luisetti, 'avvelena' i filosofi con cui si confronta (p. 12). Deleuze sottomette a tal punto Spinoza, Bergson, Nietzsche e Foucault alla 'signoria del trascendentale' da poterne ricondurre la vita e l'opera a poche 'condizioni necessarie' (p. 148). Deleuze può così immobilizzare sistematicamente ogni filosofo esaminato, facendo nascere dall'interno del suo stesso discorso un 'doppio mostruoso' che quel filosofo non riconoscerebbe mai come suo. Luisetti non fa altro che leggere Deleuze attraverso questo stesso metodo mostrando esattamente quanto egli stesso risulta 'avvelenato'

${ }^{4} \mathrm{G}$. Deleuze, L'immanenza una vita, p. 321, in Idem Due regimi di folli e altri scritti. Testi e interviste 1975-1995, Torino, Einaudi, 2010, pp. 320-324.

${ }^{5}$ G. Agamben, La potenza del pensiero, Vicenza, Neri Pozza, 2005, pp. 403-404.

${ }^{6}$ R. Esposito, Bios. Biopolitica e filosofia, cit., p. 203.

7 Deleuze si dichiara tale in Réponses à une série de questions, p. 130, in A. Villani, La guêpe et l'orchidée. Essai sur Gilles Deleuze, Paris, Belin, 1999, pp. 129-131.

${ }^{8}$ L'indeterminativo 'una' riferito alla vita è 'indice del trascendentale', L'immanenza una vita, cit., p. 322.

${ }^{9} \mathrm{G}$. Deleuze, Due regimi di folli e altri scritti. Testi e interviste 1975-1995, Torino, Einaudi, 2010, p. 255. 
dal farmakon kantiano. Una mossa interpretativa, questa di Luisetti, degna di attenzione, soprattutto per il valore strategico che assume nell'economia complessiva di Una vita. Che un 'deleuziano' possa non riconoscere il 'vero Deleuze' in quello di Luisetti, in questo caso, non solo conferma l'efficacia di questa lettura ma ne rappresenta la conseguenza più necessaria. L'approdo di Una vita è l'ipotesi, alquanto gravida di sviluppi, di una svolta naturalistica e orientalistica della biopolitica facente leva non tanto sul superamento di Deleuze quanto piuttosto su un 'prolungamento del suo movimento di pensiero lungo un'orbita eccentrica' (p. 8). Il farmakon deleuziano diventa allora antidoto e indirizza la filosofia lì dove la natura diviene con Bergson 'luogo' di un pensiero dinamico e vitale che fa uno con l'azione, mentre l'Oriente si rivela con Nietzsche l' 'impensato della nostra attualità', dunque, la 'forza impolitica' che pulsa sempre più forte nel cuore dell'Occidente (p. 87).

\section{Fabio Treppiedi}

Università Degli studi di Palermo

Dipartimento FIERI-AGLAIA

Facoltà di Lettere e Filosofia

Viale delle Scienze, Edificio 12, 90128 - Palermo (Italia)

fabiotreppiedi@gmail.com 